\title{
Loss of parkin promotes lipid rafts-dependent endocytosis through accumulating caveolin-1: implications for Parkinson's disease
}

\author{
Seon-Heui Cha ${ }^{1,2}$, Yu Ree Choi ${ }^{1,2,3}$, Cheol-Ho Heo ${ }^{4}$, Seo-Jun Kang ${ }^{1,2,3}$, Eun-Hye Joe ${ }^{1,2,3}$, Ilo Jou ${ }^{1,2,3}$,
} Hwan-Myung $\mathrm{Kim}^{4}$ and Sang Myun Park ${ }^{1,2,3^{*}}$ (D)

\begin{abstract}
Background: Parkinson's disease (PD) is characterized by progressive loss of midbrain dopaminergic neurons, resulting in motor dysfunctions. While most PD is sporadic in nature, a significant subset can be linked to either autosomal dominant or recessive mutations. PARK2, encoding the E3 ubiquitin ligase, parkin, is the most frequently mutated gene in autosomal recessive early onset PD. It has recently been reported that PD-associated gene products such as PINK1, a-synuclein, LRRK2, and DJ-1, as well as parkin associate with lipid rafts, suggesting that the dysfunction of these proteins in lipid rafts may be a causal factor of PD. Therefore here, we examined the relationship between lipid rafts-related proteins and parkin.

Results: We identified caveolin-1 (cav-1), which is one of the major constituents of lipid rafts at the plasma membrane, as a substrate of parkin. Loss of parkin function was found to disrupt the ubiquitination and degradation of cav-1, resulting in elevated cav-1 protein level in cells. Moreover, the total cholesterol level and membrane fluidity was altered by parkin deficiency, causing dysregulation of lipid rafts-dependent endocytosis. Further, cell-to-cell transmission of a-synuclein was facilitated by parkin deficiency.
\end{abstract}

Conclusions: Our results demonstrate that alterations in lipid rafts by the loss of parkin via cav-1 may be a causal factor of PD, and cav-1 may be a novel therapeutic target for PD.

Keywords: Parkin, Caveolin-1, Endocytosis, Cell-to-cell transmission

\section{Background}

Parkinson's disease (PD) is a common neurodegenerative disease characterized by progressive degeneration of midbrain dopaminergic neurons, resulting in motor dysfunction [1]. Although the majority of PD occurs sporadically, mutations in several genes including $S N C A$, LRRK2, parkin, PINK1, and DJ-1 have been identified in patients with familial PD, providing enormous insight into the molecular pathways underlying the neurodegeneration shown in PD [2]. Despite the fact that it has not yet been

\footnotetext{
* Correspondence: sangmyun@ajou.ac.kr

'Department of Pharmacology, Ajou University School of Medicine, 164, Worldcup-ro, Yeongtong-gu, Suwon 16499, Korea

${ }^{2}$ Chronic Inflammatory Disease Research Center, Ajou University School of Medicine, Suwon, Korea

Full list of author information is available at the end of the article
}

fully unveiled, oxidative stress, mitochondrial damage and defects of protein quality control system are known to play a critical role in the pathogenesis of PD [1]. Recently, prion-like propagation of $\alpha$-synuclein, encoded by $S N C A$, a $\mathrm{PD}$-associated gene, has also been proposed to play a role in the progression of PD [3-5].

Parkin is a 465 amino acid protein that possesses E3 ubiquitin ligase activity, and familial type mutations in parkin are predicted to be loss of function [6], suggesting that loss of parkin activity results in the gradual and abnormal accumulation of parkin substrates, which may cause familial PD. Mutations in the parkin gene have been revealed to be the most common cause of autosomal recessive early onset PD, which causes $50 \%$ of autosomal recessive early onset PD [7]. In 
addition, increasing evidence indicates that parkin is also inactivated in patients with sporadic PD by snitrosylation, tyrosine phosphorylation, and oxidation [8], suggesting that parkin also plays a critical role in the pathogenesis of sporadic PD. Accordingly, the identification of parkin substrates is important to elucidate the exact roles of parkin in the pathogenesis of PD. To date, several substrates including CDCrel-1 [9], Pael-R [10], synphilin-1 [11], and PARIS [12] have been identified and great efforts is continuously being made in the identification of novel substrates to elucidate the pathogenesis of PD.

Parkin is localized mainly in the cytosol, however, a small proportion has been shown to be localized in membranes including in lipid rafts [13-15]. Lipid rafts are specialized membrane microdomains that are enriched in cholesterol, glycosphingolipids, and glycosylphosphatidylinositol (GPI)-anchored proteins, which serve as organization centers for the assembly of signaling molecules, and influence membrane fluidity and trafficking of membrane proteins, and regulate receptor-mediated signal transduction and exo/endocytosis [16, 17]. Caveolins and flotillins have been shown to be integral components of lipid rafts [18]. Recently, increasing evidence has indicated that lipid rafts may play an important role in neurodegeneration $[19,20]$. In particular, many PD-associated gene products such as $\alpha$-synuclein [21], PINK1 [22], LRRK2 [23], and DJ-1 [24], as well as parkin [13], have also been reported to associate with lipid rafts, implying that functional alteration of lipid rafts by these proteins may be one of the common pathological mechanisms of PD. We recently reported that DJ-1 regulates lipid rafts-dependent endocytosis [24], however, the roles of other proteins in lipid rafts have yet to be explored.

In the present study, we explore whether parkin regulates the components of lipid rafts and whether loss of parkin induces functional alterations in lipid rafts. In addition, we explore the association between the functional alterations in lipid rafts induced by loss of parkin and the propagation of $\alpha$-synuclein, which has been recently proposed to be a causal factor of the progression of PD.

\section{Results}

Loss of parkin leads to an increase in caveolin-1 (cav-1)

To explore whether loss of parkin induces functional alterations in lipid rafts, we first compared the expression level of the well-known lipid rafts marker proteins, caveolin-1 (cav-1), cav-2, flotillin-1 (flot-1), and flot-2 using WT and parkin KO MEF cells. Interestingly, we observed that the level of cav-1 was increased in parkin $\mathrm{KO}$ MEF cells specifically, while the levels of other proteins were not changed (Fig. 1a). Confocal microscopic analysis also shows an increase of cav-1 protein in parkin KO MEF cells compared with WT MEF cells (Fig. 1b). To investigate the distribution of increased cav-1 in parkin KO MEF cells, lipid rafts were isolated based on their solubility in $1 \%$ Triton X-100 on ice [24, 25]. As shown in Fig. 1c, the increase in cav-1 protein in parkin KO MEF cells was observed only in the cold Triton X-100 insoluble fraction. Moreover, the distribution of the other proteins was not changed. The expression of parkin was relatively lower in MEF cells than that in neurons. Therefore, we loaded a 4-fold higher amount of protein for Western blotting of parkin and a small proportion of parkin was detected in the cold Triton X-100 insoluble fraction. This is also in agreement with a previous study [13]. To test whether parkin regulates cav-1 expression specifically, we overexpressed flag-parkin in parkin KO MEF cells. The overexpression of parkin in parkin KO MEF cells rescued cav-1 level without any change in the levels of other proteins (Fig. 1d), suggesting that parkin regulates cav-1 specifically.

\section{Parkin induces the degradation of cav- 1 through the proteasome-dependent pathway}

To investigate in more detail the manner in which the loss of parkin leads to an increase in cav-1, we tested the mRNA level of cav-1 in both WT and parkin KO MEF cells. Quantitative RT-PCR shows that the mRNA level of cav-1, as well as that of cav-2, flot-1, and flot-2 were not different between the two cell lines (Fig. 2a), suggesting that parkin does not regulate cav-1 at the transcriptional level. Parkin regulates protein degradation as an E3 ubiquitin ligase [6]. In addition, cav-1 has been reported to be partially degraded by the ubiquitin proteasome system (UPS) [26, 27], speculating that parkin may regulate cav-1 degradation via the UPS. To confirm this hypothesis, WT and parkin KO MEF cells were treated with cycloheximide ( $\mathrm{CHX})$, an inhibitor of protein synthesis, and the expression of cav-1 was assessed using Western blotting. As shown in Fig. 2b, the degradation of cav-1 in WT MEF cells was significantly faster than in parkin KO MEF cells. In addition, while treatment with the proteasomal inhibitors, MG-132 and lactacystin, led to an accumulation of cav-1 in WT MEF cells, it did not further enhance the steady state levels of cav-1 in parkin KO MEF cells. (Fig. 2c and d). Confocal microscopic analysis confirmed this observation (Fig. 2e), suggesting that parkin regulates cav-1 degradation via the UPS. These results also prompted speculation that cav-1 may be a substrate of parkin, thus, we tested the interaction between the two proteins. As shown in Fig. 3a, both flag-parkin and cav-1-EGFP were overexpressed in parkin KO MEF cells, and immunoprecipitation shows their interaction. Antibody staining of endogenous parkin and cav-1 in MEF cells shows the colocalization of both proteins (Fig. 3b), which was also confirmed using 


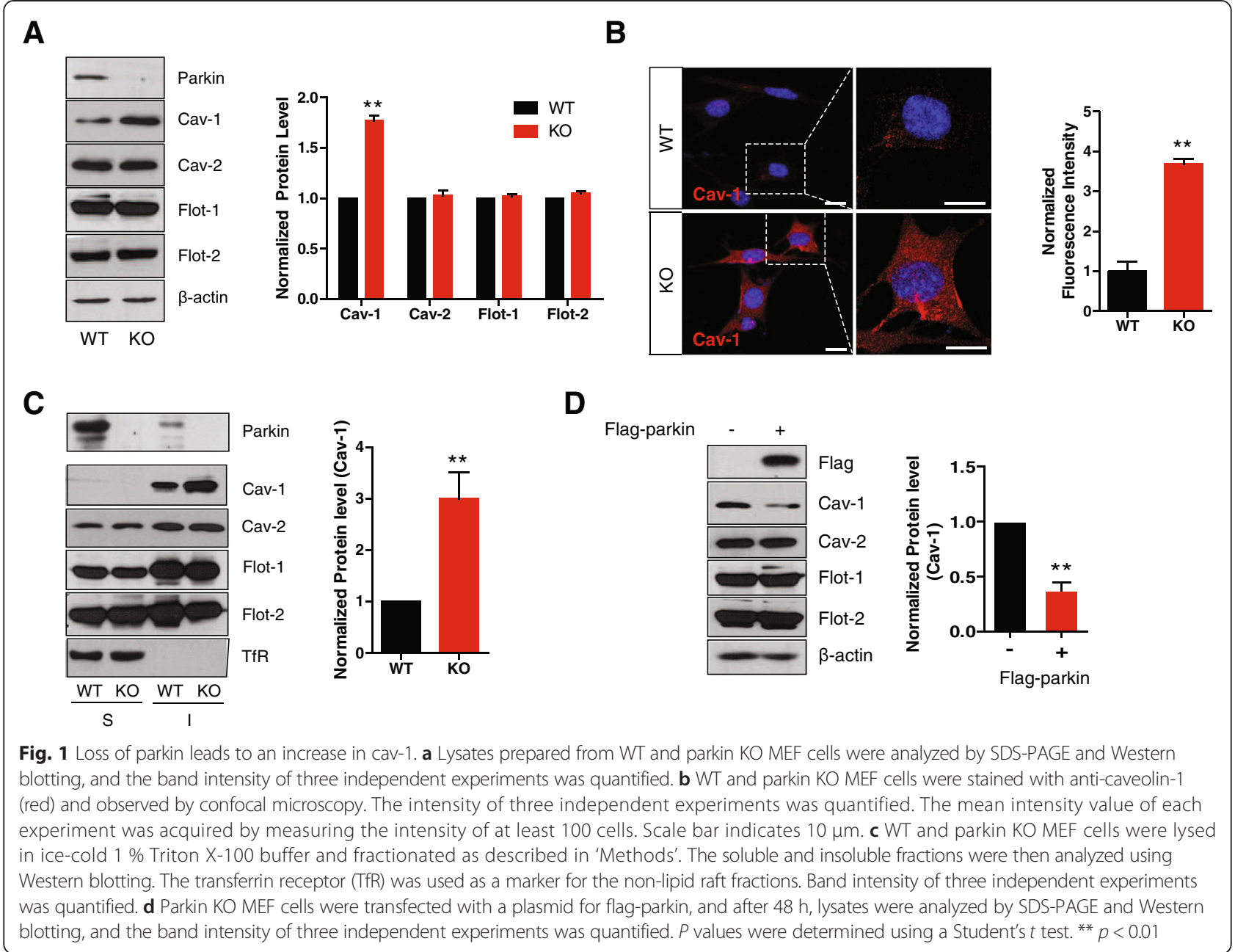

an in situ proximity ligation assay (PLA) (Fig. 3c), suggesting that parkin interacts with cav-1. Next, in order to investigate whether parkin ubiquitinates cav-1, we transfected parkin KO MEF cells with flag-parkin, His-ubiquitin, and cav-1-EGFP, which resulted in an increase in the ubiquitination of cav-1-EGFP by flagparkin (Fig. 3d). Furthermore, mutants of parkin that have been identified in patients with familial PD did not rescue the increase in cav-1 by loss of parkin (Fig. 3e), suggesting that parkin mediates the ubiquitination of cav-1, thereby targeting cav-1 to the proteasome for degradation.

\section{Cholesterol level, membrane fluidity, and lipid rafts-dependent endocytosis were altered in parkin $\mathrm{KO}$ MEF cells}

Cav-1 is known to regulate cholesterol transport [28, 29]. Accordingly, we measured the total cellular cholesterol level. As shown in Fig. 4a, the total cholesterol level in parkin KO MEF cells was slightly increased compared with that in WT MEF cells. Upon measurement of membrane fluidity using C-laurdan [30], the calculated GP value in parkin $\mathrm{KO}$ MEF cells was higher than that in WT MEF cells, suggesting that the membrane fluidity was decreased (Fig. 4b). This indicates that loss of parkin leads to an alteration in the total cholesterol level and membrane fluidity. Cav-1 has also been shown to regulate lipid rafts-dependent endocytosis [31]. In addition, the cholesterol level influences membrane fluidity, which can affect endocytosis [32]. Accordingly, in order to investigate whether parkin regulates lipid raftsdependent endocytosis via cav-1, we performed an in vitro endocytosis assay using LacCer as a marker of lipid rafts-dependent endocytosis [31, 33] and transferrin as a marker of clathrin-dependent endocytosis [34, 35]. As shown in Fig. 4c, the level of endocytosis of LacCer was higher in parkin KO MEF cells than in WT MEF cells, however, endocytosis of transferrin was not different between the two cell lines. Furthermore, to confirm that the increased level of endocytosis of LacCer in parkin $\mathrm{KO}$ MEF cells was due to the accumulation of cav-1, we downregulated cav-1 using siRNA. Transfection of cav-1 siRNA efficiently downregulated cav-1 expression (Fig. 4d), 


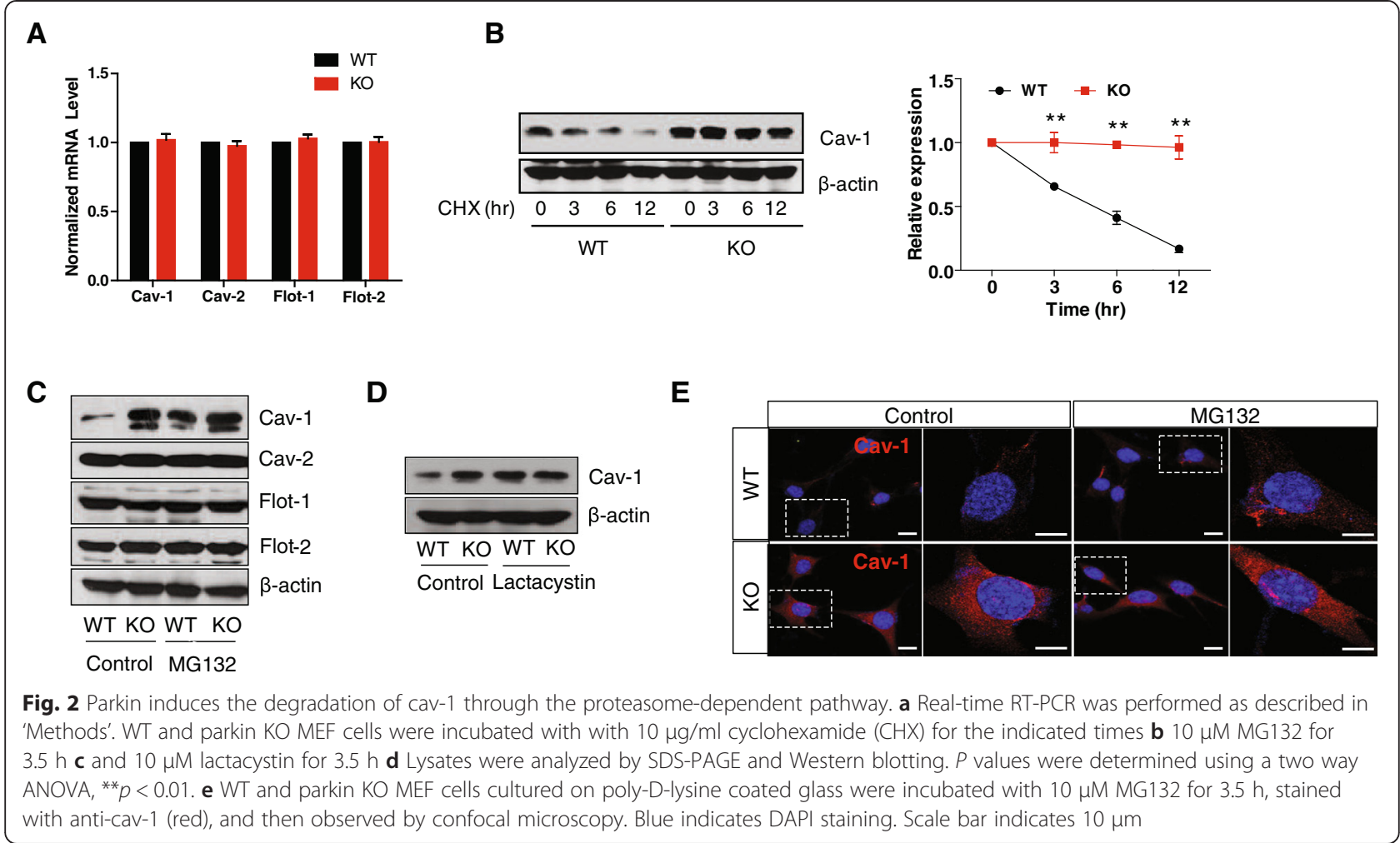

and endocytosis of LacCer in parkin KO MEF cells was rescued by the downregulation of cav-1 expression (Fig. 4c), suggesting that parkin regulates lipid raftsdependent endocytosis via cav-1.

\section{Parkin regulates lipid rafts-dependent endocytosis via cav-1 in neurons}

We performed all the experiments in MEF cells, thus, in order to confirm whether parkin regulates cav-1 in neurons, we suppressed parkin expression in primary cortical neurons using an shRNA lentiviral system. As shown in Fig. 5a, parkin level was efficiently suppressed in neuron by the shParkin lentivirus, and as a result, cav-1 expression was increased, suggesting that parkin regulates cav-1 in neurons. In addition, an in situ PLA was performed in primary cortical neurons, and the interaction of parkin with cav-1 was observed (Fig. 5b). The total cholesterol level was increased (Fig. 5c), and the membrane fluidity of parkin KD neurons was decreased compared with that of WT neurons (Fig. 5d). Moreover, the endocytosis of LacCer was enhanced by parkin knockdown (Fig. 5e), which is in agreement with our data in MEF cells (Fig. 4), suggesting that parkin regulates lipid rafts-dependent endocytosis via cav-1 in neurons.

Loss of parkin facilitates the transmission of a-synuclein Previously, we have demonstrated that $\alpha$-synuclein is internalized by lipid rafts-dependent endocytosis in
BV-2 cells, a murine microglial cell line [36]. To investigate whether parkin regulates the internalization of $\alpha$-synuclein into neurons, we used the two chamber culture system using human $\alpha$-synuclein overexpressing SH-SY5Y cells, which showed the efficient internalization of cell-released $\alpha$-synuclein into neighboring cells [36, 37]. Following staining with $\alpha$-synuclein using a human $\alpha$ synuclein-specific antibody in a co-culture of $\alpha$-synuclein overexpressing SH-SY5Y cells and rat primary cortical neurons, cell-derived $\alpha$-synuclein was observed to be internalized in much higher amounts into rat primary cortical neurons suppressing parkin expression than into control neurons (Fig. 6a), suggesting that loss of parkin could regulate the propagation of $\alpha$-synuclein. To rule out the possibility that the higher $\alpha$-synuclein intensity in rat primary cortical neurons suppressing parkin expression was due to abnormal degradation of $\alpha$-synuclein by dysfunction of parkin, we determined the endogenous $\alpha$-synuclein level following suppression of parkin expression in primary neurons. The level of endogenous $\alpha$-synuclein was not different between the primary cortical neurons infected with parkin (shParkin) and with non-targeting shRNA lentivirus (shNT) (Fig. 6b), suggesting that parkin regulates $\alpha$-synuclein transmission rather than degradation.

\section{Discussion}

Increasing evidence indicates that alteration in lipid rafts is observed in many neurodegenerative diseases. 


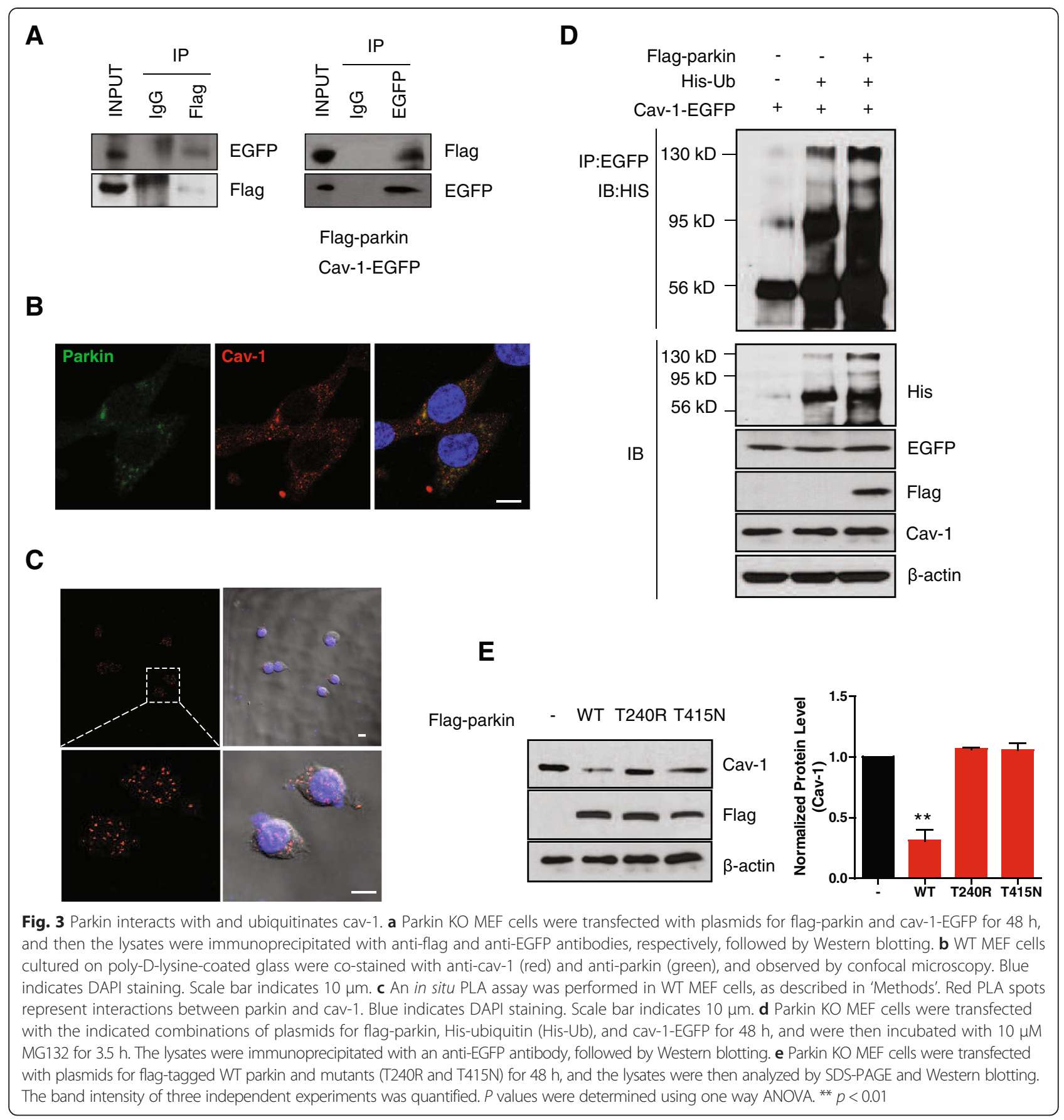

Alterations in lipid composition have been reported in lipid rafts from AD and PD human brain cortex [38, 39]. Alterations in lipid rafts proteins have also been observed in $\mathrm{AD}$ and amyotrophic lateral sclerosis mouse models $[40,41]$. Based on these observations and data from several studies, altered lipid rafts organization has also been proposed to be a common causative factor of many neurodegenerative diseases $[19,20]$.

In the present study, we identified cav-1 as a novel substrate of parkin. Cav-1 is a main structural protein component of caveolae, a subset of lipid rafts [42]. We observed that among the main structural protein components of lipid rafts, cav-1 is specifically accumulated in parkin KO MEF cells and primary neurons suppressing parkin expression. We also demonstrated that WT parkin ubiquitinates cav-1 for degradation. The Nterminal region of cav-1 has been reported to be exclusively ubiquitinated [27], suggesting that parkin can ubiquitinate any of the $\mathrm{N}$-terminal lysine residues of cav-1. On the contrary, mutations in parkin, which are 


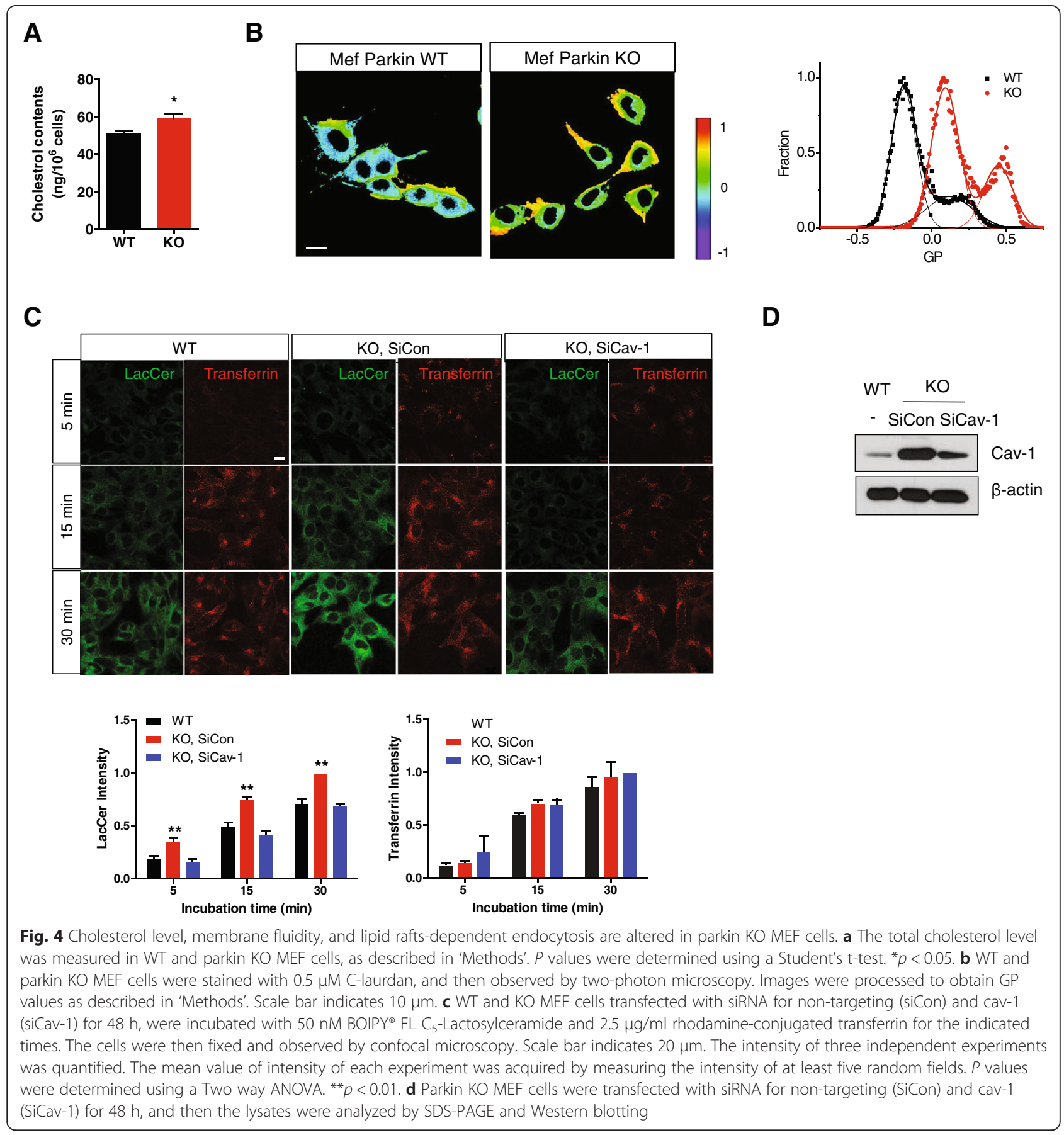

associated with familial PD, failed to regulate cav-1 expression, suggesting that accumulation of cav-1 may be involved in the pathogenesis of PD. Cav-1 is a $21-24 \mathrm{kDa}$ membrane protein, which is a member of the caveolin family consisting of cav-1, 2, and 3. Caveolins have diverse functions including membrane-initiated intracellular signaling via the clustering of proteins, the segregation of proteins, and the trafficking of proteins to and from the membrane and lipid transport [43, 44]. They are also known to be involved in diverse pathologies including carcinogenesis, insulin resistance, and aging [45]. Although the roles of cav-1 in the CNS have been relatively less studied, an association between cav-1 and neurodegeneration and aging has been reported. Cav-1 expression levels in the hippocampus are upregulated in $\mathrm{AD}$ compared with control brains [46]. An age-related increase in cav-1 has also been reported [47-49]. On the contrary, loss of cav-1 in young mice has been reported to accelerate neurodegeneration and aging [50,51]. Although the relationship between the level of cav-1 and neurodegeneration 


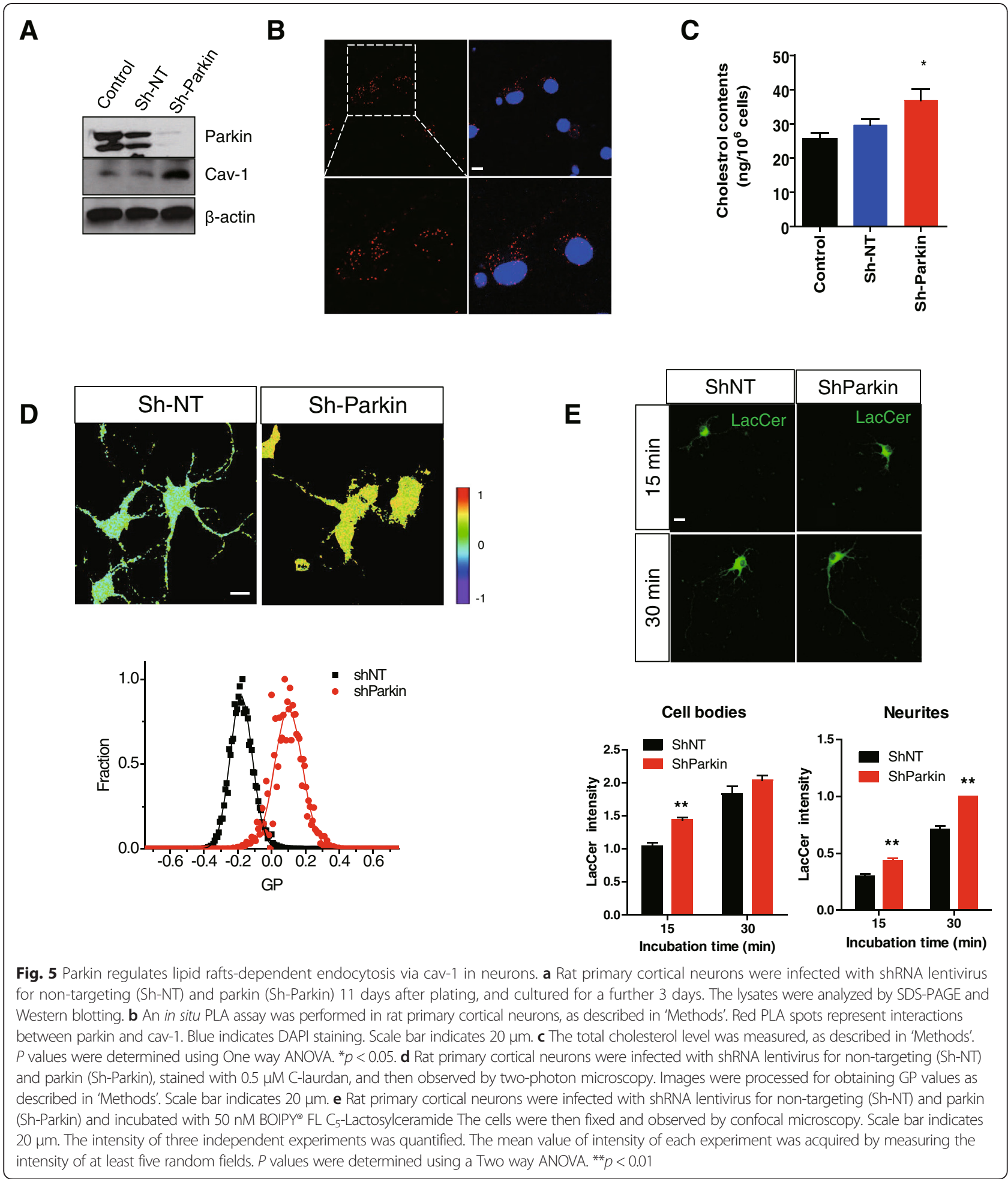

is controversial, cav-1 may be a critical control point for cellular aging [52]. Given that aging is the greatest risk factor for the development of PD [53, 54], dysregulation of cav-1 by parkin inactivation may accelerate senescence, causing neurodegeneration. Interestingly, overexpression of $\alpha$-synuclein, a PD-associated gene product, also upregulates cav-1 expression, which mediates $\alpha$-synuclein neurotoxicity $[55,56]$, supporting the involvement of the dysregulation of cav-1 in the pathogenesis of PD. 
A

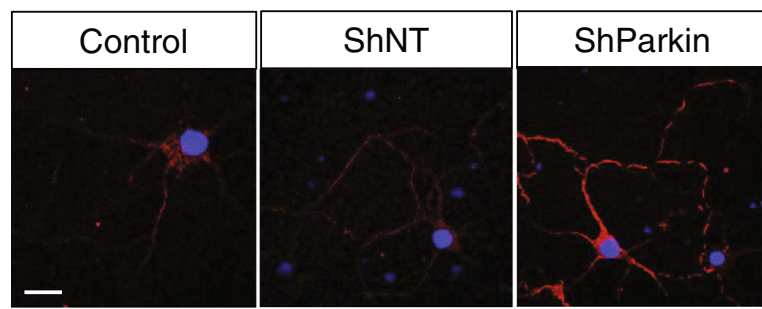

Cell bodies

Neurites
B

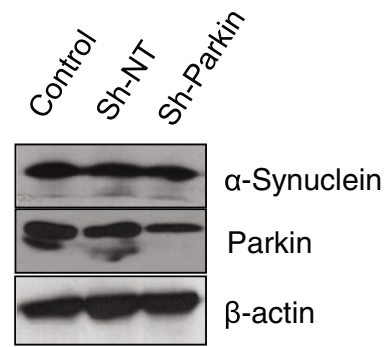

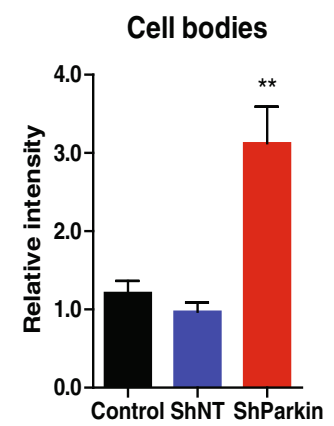

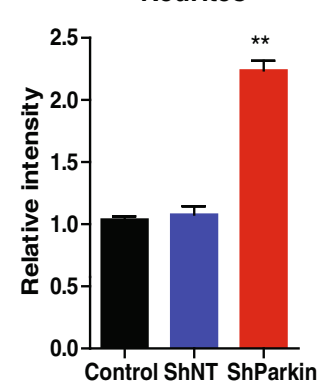

Fig. 6 Loss of parkin facilitates the transmission of a-synuclein. a Rat primary cortical neurons were infected with shRNA lentivirus for non-targeting (Sh-NT) and parkin (Sh-Parkin) 11 days after plating, cultured for a further 2 days, and then co-cultured with a-synuclein overexpressing SH-SY5Y cells for $24 \mathrm{~h}$. The cells were stained with an anti-human a-synuclein (red) antibody and observed using confocal microscopy. Blue indicates DAPI staining. Scale bar indicates $20 \mu \mathrm{m}$. b Rat primary cortical neurons were infected with shRNA lentivirus for non-targeting (Sh-NT) and parkin (Sh-Parkin) 11 days after plating and cultured for a further 3 days. The lysates were analyzed by SDS-PAGE and Western blotting

Cav-1 is well-known to play a role in the transport of cholesterol from the ER to the plasma membrane caveolae [28]. We demonstrated that accumulation of cav-1 by the loss of parkin increased the total cellular cholesterol level and decreased membrane fluidity. Upon cav-1 overexpression, total lipid cholesterol has been reported to be increased [57], which is in agreement with our study. Dysregulation of cholesterol metabolism has been largely reported to be associated with many neurodegenerative diseases including AD, PD and Huntington's disease (HD) [58]. In a model of HD, mutant htt-expressing neurons exhibited cholesterol accumulation [59] and increased cav-1 expression. Loss or reduction of cav-1 expression in a mouse model of HD suppresses the motor phenotype [60], suggesting that cav-1 is a critical factor for neurodegeneration and could be a novel therapeutic target for many neurodegenerative diseases including PD. Changes in membrane fluidity by a variety of factors, as well as the alteration of cholesterol content, may affect membrane signaling properties. It has been reported that lipid changes in AD lipid rafts increase membrane order and viscosity in these domains [61]. APP/PS1 double transgenic mice exhibit age-dependent lipid rafts changes towards higher viscosity [62]. In addition, lipid rafts in patients with $\mathrm{PD}$ are notably more viscous and liquid-ordered than age-matched controls, which may ultimately contribute to progressive neuronal dysfunction
[39], suggesting that subtle changes in membrane fluidity as a result of parkin dysfunction may affect a variety of membrane signaling, accelerating neurodegeneration.

We also demonstrated that accumulation of cav- 1 by loss of parkin increased lipid rafts-dependent endocytosis. Fallon et al. [13] reported that EGFR endocytosis is accelerated in parkin-deficient cells, however, there is no difference in transferrin endocytosis. In addition, EGFR signaling is reduced in parkin $\mathrm{KO}$ mouse brain, proposing that the interaction between parkin and Eps15 plays a role in EGFR trafficking and signaling [63]. Based on previous reports that EGFR endocytosis is lipid rafts-dependent [64], it is in agreement with our findings that parkin regulates lipid raft-dependent endocytosis. Moreover, considering previous reports that EGFR signaling is attenuated by cav-1 overexpression in senescent cells [47] and breast cancer [65], attenuated EGFR signaling shown in parkin $\mathrm{KO}$ mouse brain is speculated to be partially mediated by the accumulation of cav-1.

Recent studies have highlighted prion-like mechanisms of propagation of aggregation prone proteins including $\alpha$-synuclein, tau, and polyglutamine protein, which are associated with many neurodegenerative diseases and are anticipated to provide great insight into the pathogenesis of neurodegenerative diseases [66, 67]. In addition, numerous studies indicate functional interactions of PD-associated gene products and their 
involvement in common pathogenic mechanisms causing PD. Parkin protects against the toxicity associated with $\alpha$-synuclein [68-70]. Transgenic mice expressing a mutant parkin exhibit accumulation of proteinase Kresistant $\alpha$-synuclein [71]. GBA1 gene depletion, a strong genetic risk factor for $\mathrm{PD}$, has recently been reported to enhance cell-to-cell transmission of $\alpha$-synuclein [72]. In the present study, we observed that loss or reduction of parkin facilitated cell-to-cell transmission of $\alpha$-synuclein, speculating that dysfunction of parkin may be involved in prion-like propagation of $\alpha$-synuclein. Interestingly, compared with enhancement of lipid rafts-dependent endocytosis assessed by LacCer in parkin KD neurons, enhancement of $\alpha$-synuclein uptake was much higher in parkin $\mathrm{KD}$ neurons, indicating that other endocytic pathways may be involved in $\alpha$-synuclein uptake into neurons, and that parkin may also regulate these pathways. Parkin expression is known to be necessary for Lewy body formation [11] and parkin-mediated inclusion body formation is viewed as part of the cellular defense mechanism rather than a detrimental event $[73,74]$. Accordingly, increased $\alpha$-synuclein uptake without inclusion body formation in neurons with parkin depletion may be more detrimental. However, it has also been reported that the absence of parkin has no impact on the onset or progression of the phenotype induced by overexpression of human A53T $\alpha$-synuclein [75]. Currently, it has not been fully unveiled as to what extent prion-like propagation of $\alpha$-synuclein contributes to the pathogenesis of PD. Nevertheless, the association between the dysfunction of parkin and prion-like propagation of $\alpha$-synuclein needs to be re-evaluated.

In addition to involvement in the pathogenesis of $\mathrm{PD}$, parkin has been reported to function in metabolism $[76,77]$ and cancer [78] through the regulation of a variety of processes including receptor trafficking and mitochondrial quality control. Cav-1 has been well-reported to be associated with cancer and metabolism [79]. Accordingly, the involvement of parkin in many physiological and pathological conditions such as metabolism and cancer may be mediated by cav- 1 expression.

\section{Conclusions}

We observed the accumulation of cav-1 in parkin $\mathrm{KO}$ MEF cells and parkin KD neurons and identified cav-1 as a novel substrate of parkin. Accumulation of cav- 1 by parkin dysfunction altered the total cholesterol levels and membrane fluidity, causing alterations in lipid raftsdependent endocytosis. In addition, parkin dysfunction accelerated propagation of $\alpha$-synuclein into neighboring cells, which may contribute to the progression of PD. Accordingly, regulation of cav-1 may be a novel target to elucidate the pathogenesis of PD.

\section{Methods}

\section{Antibodies and reagents}

The following primary antibodies (Abs) were used: anti-caveoin-1 (BD bioscience, Franklin Lakes, NJ; Abcam, Cambridge, MA), anti-caveolin-2 (BD bioscience), antiflotillin-1 (BD bioscience, Franklin Lakes), anti-flotillin-2 (BD bioscience, Franklin Lakes), anti-CD71 (transferrin receptor, Santa Cruz Biotechnology, Santa Cruz, CA), anti-parkin (Santa Cruz Biotechnology, Santa Cruz, CA), anti-flag (Sigma-Aldrich, St. Louis, MO) and biotin conjugated anti-CTxB (Sigma-Aldrich, St. Louis, MO), anti-myc (Sigma-Aldrich, St. Louis, MO) and anti-actin (Santa Cruz Biotechnology, Santa Cruz, CA). MG-132 and lactacystin were purchased from Sigma-Aldrich (St. Louis, MO). Cycloheximide was purchased from Calbiochem (Denvers, $\mathrm{MA})$. Rhodamine-conjugated transferrin and BOIPY ${ }^{\circ} \mathrm{FL}$ $\mathrm{C}_{5}$-Lactosylceramide were purchased from Invitrogen/ Molecular Probes (San Dieogo, CA).

\section{Cell culture and transfection}

WT and parkin KO mouse embryonic fibroblast (MEF) cells, kindly provided by Dr. Youle RJ (National Institutes of Health, Bethesda), and human $\alpha$-synuclein overexpressing SH-SY5Y cells $[36,37]$ were grown in Dulbecco's modified Eagle's medium (DMEM) supplemented with $10 \%$ fetal bovine serum (FBS). Cells were transfected using lipofectamine 2000 or lipofectamine RNAiMax (Invitrogen, Carlsbad, CA) according to the manufacturer's instruction. After 48 or $72 \mathrm{~h}$ of transfection, cells were used for Western blotting or imaging analysis. Primary cortical neurons were cultured from SpragueDawley rat embryos at embryonic day 18 , and maintained in Neurobasal medium (Invitrogen, Carlsbad, CA) with Lglutamine and B-27 supplement (Invitrogen, Carlsbad, CA). All animal procedures used in the present study were conducted according to the guidelines established by the Ajou University School of Medicine Ethics Review Committee for Animal Experiments.

\section{Constructs}

The plasmid for flag-WT parkin was kindly provided by Dr. Chung KC (Yonsei University, Seoul), and the plasmid for His-ubiquitin was kindly provided by Dr. Park TJ (Ajou University School of Medicine, Suwon). The plasmids for parkin point mutants (T240R and T415N) were constructed using the QuickChange site-directed mutagenesis kit (Stratagene, La Jolla, CA). The plasmid for cav-1-EGFP was constructed using PCR. All constructs were verified by DNA sequencing and prepared using the Endo-Free plasmid Maxi prep Kit (Qiagen, Valencia, CA) to remove endotoxin contamination. siRNA for murine cav-1 was purchased from Bioneer (Deajeon, Korea). Lentiviral shRNA was constructed using the shRNA 
plasmid for rat cav-1 (GeneCopoeia, Rockville, MD ), as described previously [80].

\section{Western blotting and immunoprecipitation}

Cells were solubilized in lysis buffer containing $50 \mathrm{mM}$ Tris- $\mathrm{HCl}, \mathrm{pH} 7.4,150 \mathrm{mM} \mathrm{NaCl}, 0.25 \%$ sodium deoxycholate, $1 \%$ Triton X-100, and protease inhibitor mixture (GenDEPOT, Barker, TX) for $30 \mathrm{~min}$ on ice. The lysates were cleared by centrifugation at $20,000 \times \mathrm{g}$ for $15 \mathrm{~min}$ at $4{ }^{\circ} \mathrm{C}$. The supernatants were mixed with $5 \times$ sample buffer, resolved by SDS-PAGE, transferred to PVDF membrane, and analyzed by immunoblotting using the indicated antibodies. For immunoprecipitation, precleared supernatants were incubated with antibody-bound protein $\mathrm{G}$ beads (Millipore, Billerica, MA) for $6 \mathrm{~h}$ at $4{ }^{\circ} \mathrm{C}$ and washed four times with lysis buffer. Immunoprecipitates were subjected to Western blotting.

\section{Isolation of the lipid rafts fraction}

Cells were washed twice with ice-cold PBS and lysed in ice-cold PBS containing $1 \%$ Triton X-100 and protease inhibitor mixture. Following incubation for $20 \mathrm{~min}$ at $4{ }^{\circ} \mathrm{C}$, the lysates were centrifuged at $20,000 \times \mathrm{g}$ for $15 \mathrm{~min}$ at $4{ }^{\circ} \mathrm{C}$. Supernatants were used as soluble fractions. The pellets were washed with ice-cold PBS buffer, solubilized with $1 \mathrm{X}$ sample buffer and used as insoluble fractions. These individual fractions were analyzed by SDS-PAGE and Western blotting.

\section{Quantitative real-time PCR}

Total RNA was extracted from cells using Trizol reagent (Invitrogen, Carlsbad, CA) and cDNA was prepared using avian myeloblastosis virus RT (Promega, Fitchburg, WI) according to the manufacturer's instruction. cDNA samples were analyzed by the Rotor-Gene SYBR Green PCR Master mix kit on Rotor-Gene cyclers (QIAGEN, Hilden, Germany). The following primers were used: murine caveolin-1 forward, 5' - CGT AGA CTC CGA GGG ACA TC- $3^{\prime}$ and reverse, $5^{\prime}$ - TCC CTT CTG GTT CTG CAA TC -3'; murine caveolin-2; forward, 5' - CTC AAG CTA GGC TTC GAG GA-3' and reverse, 5' - GCA AGA CCA TTA GGC AGG TC - 3'; murine flotilin-1; forward, 5' - AGA AGC CTT CCA GAT GTA CC-3' and reverse, $5^{\prime}$ - ATG TCC AGT ACT TCC CCA GT - 3'; murine flotilin-2; forward, 5' - TAA GGC TGA GGC CTA CCA GA -3' and reverse, 5' - AGC AGC CGG TTT ACT TCT GA - 3'; murine actin; forward, 5' TGT TAC CAA CTG GGA CGA CA - 3' and reverse, 5'-GGG GTG TTG AAG GTC TCA AA - 3'. Gene expression was normalized to the endogenous housekeeping control gene, $\beta$-actin, which was not influenced by parkin deficiency. Relative expression was calculated for each gene using the $\Delta \Delta \mathrm{C}_{\mathrm{T}}$ (where $\mathrm{C}_{\mathrm{T}}$ is the threshold cycle) method. Statistical analysis of reverse-transcription-PCR (RT-PCR) data was based on duplicate samples.

\section{In situ proximity ligations assay (PLA)}

The PLA was performed using the DuoLink PLA kit (Sigma-Aldrich, St. Louis, MO, USA) according to the manufacturer's instructions. Briefly, cells were fixed with $4 \%$ paraformaldehyde and permeabilized with $0.1 \%$ Triton X-100. Following treatment with DuoLink blocking buffer, cells were incubated with diluted primary antibodies against parkin and cav-1. After washing, cells were incubated with species-specific PLA probes and two additional oligonucleotides under hybridization conditions. Hybridization occurs when PLA probes are in close proximity, which can be subsequently ligated to form a closed circle. A rolling-circle amplification step follows with polymerase to generate a concatemeric product, which can be visualized with fluorephore-labeled oligonucleotides after hybridization. The slides were stained with DAPI and analyzed using confocal microscopy (Zeiss, Germany).

\section{Measurement of membrane fluidity using C-laurdan}

MEF cells and primary cortical neurons cultured on polyD-lysine coated glass were incubated with cell culture media containing $0.5 \mu \mathrm{M}$ C-laurdan (SFP Co., Ltd, Chungbuk, Korea) for $20 \mathrm{~min}$ at $37^{\circ} \mathrm{C}$. The membrane fluidity was observed using two-photon fluorescence images, which were obtained with spectral confocal multiphoton microscopes (Leica TCS SP2, Wetzlar, Germany). The intensity images of C-laurdan were recorded with emissions in the range of $400-460 \mathrm{~nm}$ and $470-530 \mathrm{~nm}$, with two channels of PMTs. Generalized polarization (GP) values for each pixel were obtained using the GP analysis program, an ImageJ plugin program and were processed as described previously [30]. GP values ranged from -1 , corresponding to the highest fluidity, to +1 for the lowest fluidity.

\section{Total cellular lipid extract and quantification of cholesterol}

Total cellular lipid was extracted using the Bligh-Dyer method [81]. Briefly, $3 \mathrm{ml}$ chloroform:methanol $(2: 1, \mathrm{v} / \mathrm{v})$ was added to $1 \mathrm{ml}$ medium containing $10^{6}$ cells, followed by vigorous vortexing for $15 \mathrm{~min}$. One $\mathrm{ml}$ of $1 \mathrm{M} \mathrm{NaCl}$ was then added to the solution and vortexed for several seconds, and the mixture was centrifuged at low speed to separate the two phases $(2,000 \mathrm{rpm}$ for $15 \mathrm{~min}$ at room temperature). The upper phase was removed by a glass pipette and discarded. The interface was rinsed once with methanol:water (1:1) without mixing the whole preparation. The lower chloroform phase, containing lipids, was carefully transferred to fresh tubes and evaporated under $\mathrm{N}_{2}$ gas. The lipid residue was resuspended in 
phosphate buffer containing $0.1 \mathrm{M}$ potassium phosphate, $\mathrm{pH}$ 7.4, $50 \mathrm{mM} \mathrm{NaCl}, 5 \mathrm{mM}$ cholic acid, and $0.1 \%$ Triton $\mathrm{X}-100$. Total cholesterol levels were quantified using an Amplex Red cholesterol assay (Life Technologies, Carsbad, $\mathrm{CA}$ ) according to the manufacturer's instruction.

\section{Confocal microscopy}

Cells cultured on poly-D-lysine coated coverslips were washed twice with PBS and fixed in $4 \%$ paraformaldehyde for $30 \mathrm{~min}$ at room temperature. The fixed cells were then washed with PBS and incubated with PBS containing $0.1 \%$ Triton X-100 for $5 \mathrm{~min}$ at room temperature. After washing several times with PBS, cells were blocked with PBS containing $1 \%$ bovine serum albumin (BSA) and $0.1 \%$ Triton X-100 for $30 \mathrm{~min}$ at room temperature, and then incubated overnight with primary antibodies at $4{ }^{\circ} \mathrm{C}$. Preparations were then stained with fluorescenceconjugated secondary antibodies for $2 \mathrm{~h}$, mounted with vectashield (Vector Laboratories, Burlingame, CA), and observed under a confocal microscope (Zeiss, Germany).

\section{Statistical analysis}

All values are expressed as the mean \pm SEM. Statistical significance was evaluated using the Graphpad software (San Diego, CA).

\section{Competing interests}

The authors declare that they have no competing interests.

\section{Authors' contributions}

SHC carried out the Western blot, the immunoassays, and the PCR, participated drafted the manuscript. YRC carried out making the viral vectors. $\mathrm{CHH}$ carried out the C-Laurdan analysis, SJK carried out making the parkin mutant plasmids. HMK participated in the design of the C-Laurdan analysis. EHJ and IJ participated in its coordination. SMP performed the statistical analysis, conceived of the study, and participated in its design and coordination and prepares the manuscript. All authors read and approved the final manuscript.

\section{Acknowledgements}

The authors thank Dr. Youle RJ (National Institutes of Health, Bethesda) for providing parkin WT and KO MEFs, Dr. Chung KC (Yonsei University, Seoul, Korea) for providing the plasmid for flag-parkin, and Park TJ (Ajou University, School of Medicine, Korea) for providing the plasmid for His-ubiquitin. This work was supported by the Mid-career Research Program (2011-0016603), the MRC Program (NRF-2012R1A5A2048183), and the Basic Science Research Program (2015R1A2A2A01007457, 2014R1A1A3050501) through the National Research Foundation of Korea (NRF), funded by the Ministry of Science, ICT and future Planning.

\section{Author details \\ 'Department of Pharmacology, Ajou University School of Medicine, 164 Worldcup-ro, Yeongtong-gu, Suwon 16499, Korea. ${ }^{2}$ Chronic Inflammatory Disease Research Center, Ajou University School of Medicine, Suwon, Korea. ${ }^{3}$ Neuroscience Graduate Program, Ajou University School of Medicine, Suwon, Korea. ${ }^{4}$ Department of Chemistry, Ajou University, Suwon, Korea.}

Received: 25 September 2015 Accepted: 23 November 2015 Published online: 01 December 2015

\section{References}

1. Lees AJ, Hardy J, Revesz T. Parkinson's disease. Lancet. 2009;373:2055-66.

2. Corti O, Lesage S, Brice A. What genetics tells us about the causes and mechanisms of Parkinson's disease. Physiol Rev. 2011;91:1161-218.
3. Desplats P, Lee HJ, Bae EJ, Patrick C, Rockenstein E, Crews L, et al. Inclusion formation and neuronal cell death through neuron-to-neuron transmission of alpha-synuclein. Proc Natl Acad Sci U S A. 2009;106:13010-5.

4. Luk KC, Kehm V, Carroll J, Zhang B, O'Brien P, Trojanowski JQ, et al. Pathological alpha-synuclein transmission initiates Parkinson-like neurodegeneration in nontransgenic mice. Science. 2012;338:949-53.

5. Volpicelli-Daley LA, Luk KC, Patel TP, Tanik SA, Riddle DM, Stieber A, et al. Exogenous alpha-synuclein fibrils induce Lewy body pathology leading to synaptic dysfunction and neuron death. Neuron. 2011;72:57-71.

6. Shimura H, Hattori N, Kubo S, Mizuno Y, Asakawa S, Minoshima S, et al. Familial Parkinson disease gene product, parkin, is a ubiquitin-protein ligase. Nat Genet. 2000;25:302-5.

7. Puschmann A. Monogenic Parkinson's disease and parkinsonism: clinical phenotypes and frequencies of known mutations. Parkinsonism Relat Disord. 2013;19:407-15.

8. Dawson TM, Dawson VL. Parkin plays a role in sporadic Parkinson's disease. Neurodegener Dis. 2014;13:69-71

9. Zhang Y, Gao J, Chung KK, Huang H, Dawson VL, Dawson TM. Parkin functions as an E2-dependent ubiquitin- protein ligase and promotes the degradation of the synaptic vesicle-associated protein, CDCrel-1. Proc Natl Acad Sci U S A. 2000;97:13354-9.

10. Imai Y, Soda M, Inoue H, Hattori N, Mizuno Y, Takahashi R. An unfolded putative transmembrane polypeptide, which can lead to endoplasmic reticulum stress, is a substrate of Parkin. Cell. 2001;105:891-902.

11. Chung KK, Zhang Y, Lim KL, Tanaka Y, Huang H, Gao J, et al. Parkin ubiquitinates the alpha-synuclein-interacting protein, synphilin-1: implications for Lewy-body formation in Parkinson disease. Nat Med. 2001;7:1144-50.

12. Shin JH, Ko HS, Kang H, Lee Y, Lee Yl, Pletinkova O, et al. PARIS (ZNF746) repression of PGC-1alpha contributes to neurodegeneration in Parkinson's disease. Cell. 2011;144:689-702.

13. Fallon L, Moreau F, Croft BG, Labib N, Gu WJ, Fon EA. Parkin and CASK/LIN-2 associate via a PDZ-mediated interaction and are co-localized in lipid rafts and postsynaptic densities in brain. J Biol Chem. 2002;277:486-91.

14. Shimura $\mathrm{H}$, Hattori $\mathrm{N}$, Kubo $\mathrm{S}$, Yoshikawa M, Kitada T, Matsumine $\mathrm{H}$, et al. Immunohistochemical and subcellular localization of Parkin protein: absence of protein in autosomal recessive juvenile Parkinsonism patients. Ann Neurol. 1999:45:668-72.

15. Kubo SI, Kitami T, Noda S, Shimura H, Uchiyama Y, Asakawa S, et al. Parkin is associated with cellular vesicles. J Neurochem. 2001;78:42-54.

16. Korade Z, Kenworthy AK. Lipid rafts, cholesterol, and the brain. Neuropharmacology. 2008;55:1265-73.

17. Simons K, Ikonen E. Functional rafts in cell membranes. Nature. 1997;387: 569-72.

18. Quest AF, Leyton L, Parraga M. Caveolins, caveolae, and lipid rafts in cellular transport, signaling, and disease. Biochem Cell Biol. 2004;82:129-44.

19. Schengrund CL. Lipid rafts: keys to neurodegeneration. Brain Res Bull. 2010; 82:7-17.

20. Sonnino S, Aureli M, Grassi S, Mauri L, Prioni S, Prinetti A. Lipid Rafts in Neurodegeneration and Neuroprotection. Mol Neurobiol. 2014;50:130-48.

21. Fortin DL, Troyer MD, Nakamura K, Kubo S, Anthony MD, Edwards RH. Lipid rafts mediate the synaptic localization of alpha-synuclein. J Neurosci. 2004; 24:6715-23.

22. Silvestri L, Caputo V, Bellacchio E, Atorino L, Dallapiccola B, Valente EM, et al. Mitochondrial import and enzymatic activity of PINK1 mutants associated to recessive Parkinsonism. Hum Mol Genet. 2005;14:3477-92.

23. Hatano T, Kubo S, Imai S, Maeda M, Ishikawa K, Mizuno Y, et al. Leucine-rich repeat kinase 2 associates with lipid rafts. Hum Mol Genet. 2007;16:678-90.

24. Kim KS, Kim JS, Park JY, Suh YH, Jou I, Joe EH, et al. DJ-1 associates with lipid rafts by palmitoylation and regulates lipid rafts-dependent endocytosis in astrocytes. Hum Mol Genet. 2013;22:4805-17.

25. Zlatkine P, Mehul B, Magee Al. Retargeting of cytosolic proteins to the plasma membrane by the Lck protein tyrosine kinase dual acylation motif. J Cell Sci. 1997;110(Pt 5):673-9.

26. Hayer A, Stoeber M, Ritz D, Engel S, Meyer HH, Helenius A. Caveolin-1 is ubiquitinated and targeted to intralumenal vesicles in endolysosomes for degradation. J Cell Biol. 2010;191:615-29.

27. Kirchner $\mathrm{P}, \mathrm{Bug} \mathrm{M}, \mathrm{Meyer} \mathrm{H}$. Ubiquitination of the $\mathrm{N}$-terminal region of caveolin-1 regulates endosomal sorting by VCP/p97. J Biol Chem. 2013;288:7363-72.

28. Ikonen E, Parton RG. Caveolins and cellular cholesterol balance. Traffic. 2000; $1: 212-7$. 
29. Smart EJ, Ying Y, Donzell WC, Anderson RG. A role for caveolin in transport of cholesterol from endoplasmic reticulum to plasma membrane. J Biol Chem. 1996;271:29427-35.

30. Kim HM, Choo HJ, Jung SY, Ko YG, Park WH, Jeon SJ, et al. A two-photon fluorescent probe for lipid raft imaging: C-laurdan. Chembiochem. 2007;8:553-9.

31. Singh RD, Puri V, Valiyaveettil JT, Marks DL, Bittman R, Pagano RE. Selective caveolin-1-dependent endocytosis of glycosphingolipids. Mol Biol Cell. 2003; 14:3254-65.

32. Spector AA, Yorek MA. Membrane lipid composition and cellular function. J Lipid Res. 1985;26:1015-35.

33. Marks DL, Singh RD, Choudhury A, Wheatley CL, Pagano RE. Use of fluorescent sphingolipid analogs to study lipid transport along the endocytic pathway. Methods. 2005;36:186-95.

34. Maxfield FR, McGraw TE. Endocytic recycling. Nat Rev Mol Cell Biol. 2004;5: 121-32.

35. Widera A, Norouziyan F, Shen WC. Mechanisms of TfR-mediated transcytosis and sorting in epithelial cells and applications toward drug delivery. Adv Drug Deliv Rev. 2003;55:1439-66.

36. Park JY, Kim KS, Lee SB, Ryu JS, Chung KC, Choo YK, et al. On the mechanism of internalization of alpha-synuclein into microglia: roles of ganglioside GM1 and lipid raft. J Neurochem. 2009;110:400-11.

37. Kim KS, Choi YR, Park JY, Lee JH, Kim DK, Lee SJ, et al. Proteolytic cleavage of extracellular alpha-synuclein by plasmin: implications for Parkinson disease. J Biol Chem. 2012;287:24862-72.

38. Martin V, Fabelo N, Santpere G, Puig B, Marin R, Ferrer I, et al. Lipid alterations in lipid rafts from Alzheimer's disease human brain cortex. J Alzheimers Dis. 2010;19:489-502.

39. Fabelo N, Martin V, Santpere G, Marin R, Torrent L, Ferrer I, et al. Severe alterations in lipid composition of frontal cortex lipid rafts from Parkinson's disease and incidental Parkinson's disease. Mol Med. 2011;17:1107-18.

40. Chadwick W, Brenneman R, Martin B, Maudsley S. Complex and multidimensional lipid raft alterations in a murine model of Alzheimer's disease. Int J Alzheimers Dis. 2010;2010:604792.

41. Zhai J, Strom AL, Kilty R, Venkatakrishnan P, White J, Everson WV, et al. Proteomic characterization of lipid raft proteins in amyotrophic lateral sclerosis mouse spinal cord. FEBS J. 2009:276:3308-23.

42. Lajoie P, Nabi IR. Lipid rafts, caveolae, and their endocytosis. Int Rev Cell Mol Biol. 2010;282:135-63.

43. Stern CM, Mermelstein PG. Caveolin regulation of neuronal intracellular signaling. Cell Mol Life Sci. 2010;67:3785-95.

44. Liu P, Rudick M, Anderson RG. Multiple functions of caveolin-1. J Biol Chem. 2002;277:41295-8

45. Cohen AW, Hnasko R, Schubert W, Lisanti MP. Role of caveolae and caveolins in health and disease. Physiol Rev. 2004;84:1341-79.

46. Gaudreault SB, Dea D, Poirier J. Increased caveolin-1 expression in Alzheimer's disease brain. Neurobiol Aging. 2004;25:753-9.

47. Park WY, Park JS, Cho KA, Kim DI, Ko YG, Seo JS, et al. Up-regulation of caveolin attenuates epidermal growth factor signaling in senescent cells. J Biol Chem. 2000;275:20847-52.

48. Kang MJ, Chung YH, Hwang Cl, Murata M, Fujimoto T, Mook-Jung $\mid H_{\text {, et al }}$ Caveolin-1 upregulation in senescent neurons alters amyloid precursor protein processing. Exp Mol Med. 2006;38:126-33.

49. Volonte D, Zhang K, Lisanti MP, Galbiati F. Expression of caveolin-1 induces premature cellular senescence in primary cultures of murine fibroblasts. Mol Biol Cell. 2002;13:2502-17.

50. Head BP, Peart JN, Panneerselvam M, Yokoyama T, Pearn ML, Niesman IR, et al. Loss of caveolin-1 accelerates neurodegeneration and aging. PLOS One. 2010:5:e15697.

51. Park DS, Cohen AW, Frank PG, Razani B, Lee H, Williams TM, et al. Caveolin-1 null (-/-) mice show dramatic reductions in life span. Biochemistry. 2003;42: 15124-31

52. Cho KA, Park SC. Caveolin-1 as a prime modulator of aging: a new modality for phenotypic restoration? Mech Ageing Dev. 2005;126:105-10.

53. Collier TJ, Kanaan NM, Kordower JH. Ageing as a primary risk factor for Parkinson's disease: evidence from studies of non-human primates. Nat Rev Neurosci. 2011:12:359-66.

54. Rodriguez M, Rodriguez-Sabate C, Morales I, Sanchez A, Sabate M. Parkinson's disease as a result of aging. Aging Cell. 2015;14:293-308.

55. Madeira A, Yang J, Zhang X, Vikeved E, Nilsson A, Andren PE, et al. Caveolin-1 interacts with alpha-synuclein and mediates toxic actions of cellular alpha-synuclein overexpression. Neurochem Int. 2011;59:280-9.
56. Hashimoto M, Takenouchi T, Rockenstein E, Masliah E. Alpha-synuclein up-regulates expression of caveolin-1 and down-regulates extracellular signal-regulated kinase activity in B103 neuroblastoma cells: role in the pathogenesis of Parkinson's disease. J Neurochem. 2003;85:1468-79.

57. Toselli M, Biella G, Taglietti V, Cazzaniga E, Parenti M. Caveolin-1 expression and membrane cholesterol content modulate $\mathrm{N}$-type calcium channel activity in NG108-15 cells. Biophys J. 2005;89:2443-57.

58. Martin MG, Pfrieger F, Dotti CG. Cholesterol in brain disease: sometimes determinant and frequently implicated. EMBO Rep. 2014;15:1036-52.

59. Trushina E, Singh RD, Dyer RB, Cao S, Shah VH, Parton RG, et al. Mutant huntingtin inhibits clathrin-independent endocytosis and causes accumulation of cholesterol in vitro and in vivo. Hum Mol Genet. 2006;15: 3578-91.

60. Trushina E, Canaria CA, Lee DY, McMurray CT. Loss of caveolin-1 expression in knock-in mouse model of Huntington's disease suppresses pathophysiology in vivo. Hum Mol Genet. 2014;23:129-44.

61. Fabelo N, Martin V, Marin R, Moreno D, Ferrer I, Diaz M. Altered lipid composition in cortical lipid rafts occurs at early stages of sporadic Alzheimer's disease and facilitates APP/BACE1 interactions. Neurobiol Aging. 2014;35:1801-12

62. Fabelo N, Martin V, Marin R, Santpere G, Aso E, Ferrer I, et al. Evidence for premature lipid raft aging in APP/PS1 double-transgenic mice, a model of familial Alzheimer disease. J Neuropathol Exp Neurol. 2012;11:868-81.

63. Fallon L, Belanger CM, Corera AT, Kontogiannea M, Regan-Klapisz E, Moreau $F$, et al. A regulated interaction with the UIM protein Eps15 implicates parkin in EGF receptor trafficking and PI(3)K-Akt signalling. Nat Cell Biol. 2006:8:834-42

64. Sigismund S, Woelk T, Puri C, Maspero E, Tacchetti C, Transidico P, et al. Clathrin-independent endocytosis of ubiquitinated cargos. Proc Natl Acad Sci U S A. 2005:102:2760-5.

65. Agelaki S, Spiliotaki M, Markomanolaki H, Kallergi G, Mavroudis D, Georgoulias V, et al. Caveolin-1 regulates EGFR signaling in MCF-7 breast cancer cells and enhances gefitinib-induced tumor cell inhibition. Cancer Biol Ther. 2009:8:1470-7.

66. Frost B, Diamond MI. Prion-like mechanisms in neurodegenerative diseases. Nat Rev Neurosci. 2010:11:155-9.

67. Goedert M, Clavaguera F, Tolnay M. The propagation of prion-like protein inclusions in neurodegenerative diseases. Trends Neurosci. 2010;33:317-25.

68. Petrucelli L, O'Farrell C, Lockhart PJ, Baptista M, Kehoe K, Vink L, et al. Parkin protects against the toxicity associated with mutant alpha-synuclein: proteasome dysfunction selectively affects catecholaminergic neurons. Neuron. 2002;36:1007-19.

69. Oluwatosin-Chigbu Y, Robbins A, Scott CW, Arriza JL, Reid JD, Zysk JR. Parkin suppresses wild-type alpha-synuclein-induced toxicity in SHSY-5Y cells. Biochem Biophys Res Commun. 2003;309:679-84.

70. Lo Bianco C, Schneider BL, Bauer M, Sajadi A, Brice A, Iwatsubo T, et al. Lentiviral vector delivery of parkin prevents dopaminergic degeneration in an alpha-synuclein rat model of Parkinson's disease. Proc Natl Acad Sci USA. 2004:101:17510-5.

71. Lu XH, Fleming SM, Meurers B, Ackerson LC, Mortazavi F, Lo V, et al. Bacterial artificial chromosome transgenic mice expressing a truncated mutant parkin exhibit age-dependent hypokinetic motor deficits, dopaminergic neuron degeneration, and accumulation of proteinase K-resistant alpha-synuclein. J Neurosci. 2009;29:1962-76.

72. Bae EJ, Yang NY, Song M, Lee CS, Lee JS, Jung BC, et al. Glucocerebrosidase depletion enhances cell-to-cell transmission of alpha-synuclein. Nat Commun. 2014:5:4755

73. Lotharius J, Brundin P. Pathogenesis of Parkinson's disease: dopamine vesicles and alpha-synuclein. Nat Rev Neurosci. 2002:3:932-42.

74. Ciechanover A, Brundin P. The ubiquitin proteasome system in neurodegenerative diseases: sometimes the chicken, sometimes the egg Neuron. 2003:40:427-46.

75. von Coelln R, Thomas B, Andrabi SA, Lim KL, Savitt JM, Saffary R, et al. Inclusion body formation and neurodegeneration are parkin independent in a mouse model of alpha-synucleinopathy. J Neurosci. 2006;26:3685-96.

76. Kim KY, Stevens MV, Akter MH, Rusk SE, Huang RJ, Cohen A, et al. Parkin is a lipid-responsive regulator of fat uptake in mice and mutant human cells. J Clin Invest. 2011;121:3701-12.

77. Zhang C, Lin M, Wu R, Wang X, Yang B, Levine AJ, et al. Parkin, a p53 target gene, mediates the role of p53 in glucose metabolism and the Warburg effect. Proc Natl Acad Sci U S A. 2011;108:16259-64. 
78. Gong Y, Zack TI, Morris LG, Lin K, Hukkelhoven E, Raheja R, et al. Pan-cancer genetic analysis identifies PARK2 as a master regulator of $\mathrm{G} 1 / \mathrm{S}$ cyclins. Nat Genet. 2014;46:588-94.

79. Sotgia F, Martinez-Outschoorn UE, Howell A, Pestell RG, Pavlides S, Lisanti MP. Caveolin-1 and cancer metabolism in the tumor microenvironment: markers, models, and mechanisms. Annu Rev Pathol. 2012;7:423-67.

80. Torashima T, Okoyama S, Nishizaki T, Hirai H. In vivo transduction of murine cerebellar Purkinje cells by HIV-derived lentiviral vectors. Brain Res. 2006; 1082:11-22.

81. Dibya D, Arora N, Smith EA. Noninvasive measurements of integrin microclustering under altered membrane cholesterol levels. Biophys J. 2010; 99:853-61.

Submit your next manuscript to BioMed Central and we will help you at every step:

- We accept pre-submission inquiries

- Our selector tool helps you to find the most relevant journal

- We provide round the clock customer support

- Convenient online submission

- Thorough peer review

- Inclusion in PubMed and all major indexing services

- Maximum visibility for your research 